\title{
Transcriptional activation of integrin $\beta 6$ during the epithelial-mesenchymal transition defines a novel prognostic indicator of aggressive colon carcinoma
}

\author{
Richard C. Bates, ${ }^{1}$ David I. Bellovin, ${ }^{1}$ Courtney Brown, ${ }^{2}$ Elizabeth Maynard, ${ }^{2}$ Bingyan Wu, ${ }^{3}$ \\ Hisaaki Kawakatsu, ${ }^{4}$ Dean Sheppard, ${ }^{4}$ Peter Oettgen, ${ }^{2}$ and Arthur M. Mercurio ${ }^{1}$

\begin{abstract}
1Division of Cancer Biology and Angiogenesis, Department of Pathology, and 2Division of Cardiology, Beth Israel Deaconess Medical Center and Harvard Medical School, Boston, Massachusetts, USA. ${ }^{3}$ Department of Biostatistical Science, Dana-Farber Cancer Institute, Boston, Massachusetts, USA. ${ }^{4}$ Lung Biology Center and Department of Medicine, UCSF, San Francisco, California, USA.
\end{abstract}

\begin{abstract}
We used a spheroid model of colon carcinoma to analyze integrin dynamics as a function of the epithelialmesenchymal transition (EMT), a process that provides a paradigm for understanding how carcinoma cells acquire a more aggressive phenotype. This EMT involves transcriptional activation of the $\beta 6$ integrin subunit and a consequent induction of $\alpha v \beta 6$ expression. This integrin enhances the tumorigenic properties of colon carcinoma, including activation of autocrine TGF- $\beta$ and migration on interstitial fibronectin. Importantly, this study validates the clinical relevance of the EMT. Kaplan-Meier analysis of $\beta 6$ expression in 488 colorectal carcinomas revealed a striking reduction in median survival time of patients with high $\beta 6$ expression. Elevated receptor expression did not simply reflect increasing tumor stage, since log-rank analysis showed a more significant impact on the survival of patients with early-stage, as opposed to late-stage, disease. Cox regression analysis confirmed that this integrin is an independent variable for these tumors. These findings define the $\alpha v \beta 6$ integrin as an important risk factor for early-stage disease and a novel therapeutic candidate for colorectal cancer.
\end{abstract}

\section{Introduction}

A dynamic transition of epithelia into mesenchyme occurs during normal morphogenetic processes such as embryonic development, tissue remodeling, and wound repair by a process known as the epithelial-mesenchymal transition (EMT; reviewed in refs. 1-3). Of particular interest to cancer biologists is the emerging realization that the progression of epithelial-derived tumors (carcinomas) may also involve spatial or temporal occurrences of EMT (4-7), allowing carcinoma cells to acquire a more aggressive phenotype. This hypothesis is supported by the observation that molecules whose expression is altered during the EMT, such as E-cadherin, are often useful markers for prognosis. The mechanism of the EMT is complex and involves signals from the microenvironment, such as TGF- $\beta$, a well-characterized inducer of the process in a variety of in vitro and in vivo settings $(5,6)$. Although loss of E-cadherin may be a primal event for EMT, alterations in other adhesion mechanisms must occur to generate a mesenchymal phenotype. In this respect, the paradigm of an EMT is apt because an invasive carcinoma cell must acquire the ability to interact with distinct interstitial matrices subsequent to its transgression of the basement membrane. Accordingly, the integrin family of adhesion molecules represents the major receptors that mediate attachment to the ECM, with ligand occupancy triggering critical intracellular signaling pathways $(8,9)$. Surprisingly, however, little is known about how integrin expression and function are regulated during EMT.

Nonstandard abbreviations used: EMT, epithelial-mesenchymal transition. Conflict of interest: The authors have declared that no conflict of interest exists.

Citation for this article: J. Clin. Invest. 115:339-347 (2005)

doi:10.1172/JCI200523183.
Here, we have used our recently characterized in vitro model of EMT in colon carcinoma, which employs a multicellular, 3D spheroid cell system (10), to analyze integrin dynamics as a function of EMT. We report that the EMT induces a marked increase in the expression of the $\alpha v \beta 6$ integrin, a receptor for fibronectin and tenascin. This increase is dependent on the transcriptional activation of the $\beta 6$ integrin subunit mediated by Ets-1, a result that implicates this proto-oncogene as a key regulator of EMT. Further, the consequences of elevated $\alpha v \beta 6$ expression are directly linked to both the mechanism of the EMT itself and the function of mesenchymal cells. The $\alpha v \beta 6$ integrin promotes the activation of autocrine TGF- $\beta$ that sustains the EMT and is also required for the migration of post-EMT cells on fibronectin. Moreover, this study validates our EMT model as a valuable tool for the identification of clinically relevant markers, as analysis of almost 500 colorectal carcinoma samples revealed that tumors with elevated $\alpha v \beta 6$ expression are associated with a significantly reduced survival time of patients in comparison with tumors with no $\beta 6$ expression or low $\beta 6$ expression. Finally, our results define $\beta 6$ expression as an independent prognostic variable for colorectal cancer and, most significantly, one that is predictive of outcome in early-stage disease.

\section{Results}

Increased expression of $\alpha v \beta 6$ integrin accompanies EMT of LIM 1863 organoids. We recently characterized a novel colon carcinoma model for EMT, in which the well-differentiated LIM 1863 cell line switches from a suspension culture of $3 \mathrm{D}$ spheroids (termed organoids) to a migratory monolayer phenotype following exposure to TGF- $\beta$ (10). Further, we demonstrated that this bona fide EMT conversion was accelerated in response to a synergistic effect of TNF- $\alpha$ signaling (10). LIM 1863 organoids express $2 \alpha v$ 

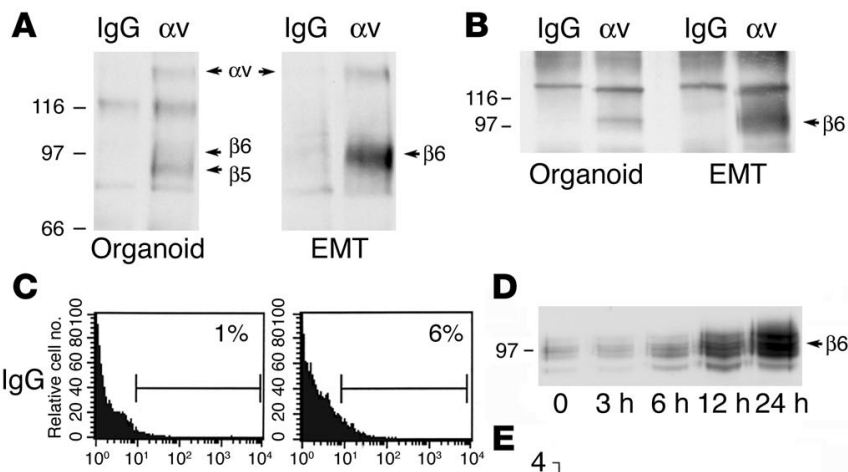

D
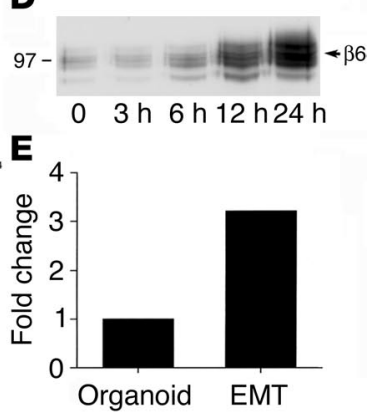

\section{Figure 1}

Integrin $\alpha v \beta 6$ expression increases following EMT. (A) LIM 1863 organoids or cells harvested 24 hours after induction of EMT were surface biotinylated, and the extracts were immunoprecipitated with an mAb directed against integrin $\alpha v$ or control IgG. Relative molecular masses are shown to the left in $\mathrm{kDa}$. Arrows indicate the positions of $\alpha \mathrm{V}$, and its associated chains $\beta 5$ and $\beta 6$. (B) Cell extracts were prepared from organoids or from cells 24 hours after EMT, immunoprecipitated as in $\mathbf{A}$, and then immunoblotted with an mAb against the $\beta 6$ integrin subunit. Relative molecular masses are shown to the left in $\mathrm{kDa}$. (C) LIM 1863 organoids were either disaggregated into single-cell suspensions (Organoid) or harvested 24 hours after induction of the EMT (EMT), and surface expression of $\beta 6$ was assessed by flow cytometry. A greater-than-3-fold increase in $\beta 6$ surface expression occurs after the EMT. (D) Cell extracts were prepared over the time course shown after EMT induction and immunoblotted with an anti- $\beta 6$ antibody to determine the kinetics of upregulation of the receptor. Relative molecular masses are shown to the left in $\mathrm{kDa}$. (E) Integrin $\beta 6$ mRNA levels were quantified using real-time quantitative PCR in organoid or EMT cultures of LIM 1863 cells. The fold change between treatments (3.2-fold induction following the EMT) is represented graphically.

The time course of $\beta 6$ induction during the EMT was evaluated by immunoblotting (Figure 1D). Expression remained low during the first 3 hours, with an initial increase apparent at 6-12 hours and maximal expression seen at 24 hours. In contrast, expression of another cell surface receptor, the $\alpha 6 \beta 4$ integrin, remained unchanged over the same time course (data not shown). We then sought to determine whether this $\beta 6$ upregulation occurs at the mRNA level using real-time quantitative PCR. As shown in Figure $1 \mathrm{E}$, we observed a greater-than-3-fold increase in mRNA level for $\beta 6$ in post-EMT cells as compared with organoids, a result consistent with the flow cytometry data. Taken together, the data

\section{Figure 2}

Transcriptional regulation of $\beta 6$ by the Ets-1 transcription factor. (A) Schematic of the human integrin $\beta 6$ promoter. The transcription start site (TSS) and translation start site (ATG) are indicated. Putative Ets-binding sites are shown (triangles), and the 4 corresponding sequences are listed, including location detail. The consensus DNA-binding sequence (GGAA) is shown in bold. (B) Transactivation of the $\beta 6$ luciferase reporter construct $(-926 /+208)$ by a panel of Ets transcription factors compared with the empty mammalian expression plasmid (PCI) in HEK293 cells. The change in luciferase activity is expressed as fold induction compared with $\mathrm{PCl}$. (C) Gel mobility shift assay for Ets-1 binding to putative Ets sites in the $\beta 6$ promoter. In vitro-translated Ets-1 protein or control extract was used with endlabeled oligonucleotide probes encoding putative Ets sites 1-4, as indicated. (D) Mutational analysis of the $\beta 6$ promoter. Transactivation of either the wild-type (black bars) or mutant Ets-1-binding site $-66 /-63$ (white bars) $\beta 6$ luciferase reporter constructs in response to increasing doses of Ets-1 is shown.

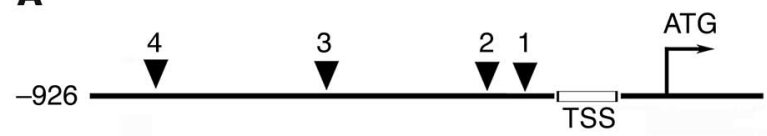

B

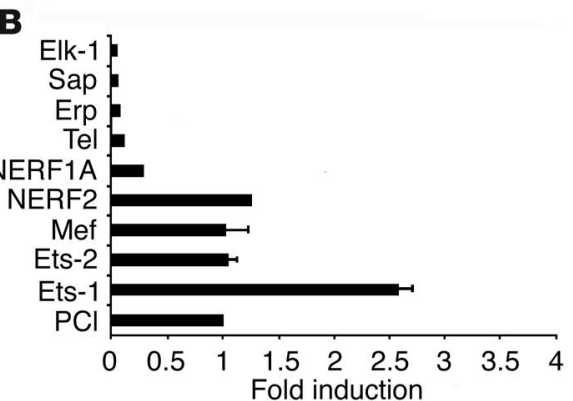

C

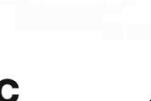

Site 1:-69 CAA GGAA GTA -60 Site 2: -79 TAA GGAA GGC -88 Site 3:-422 GAA GGAA GGC -431 Site 4:-804 TCA GGAA GGT -813

\section{D}

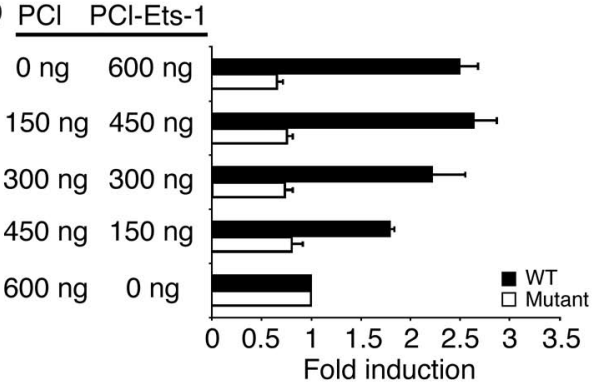

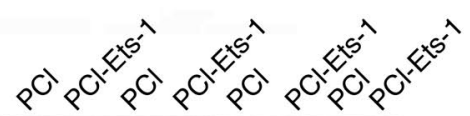
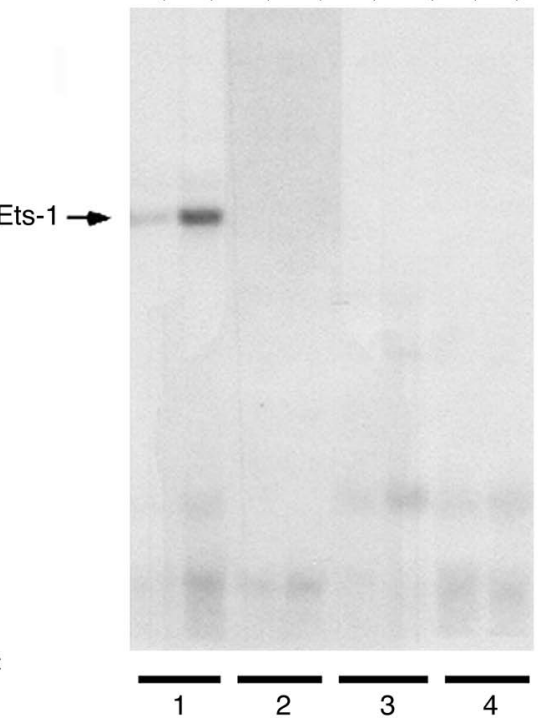
A
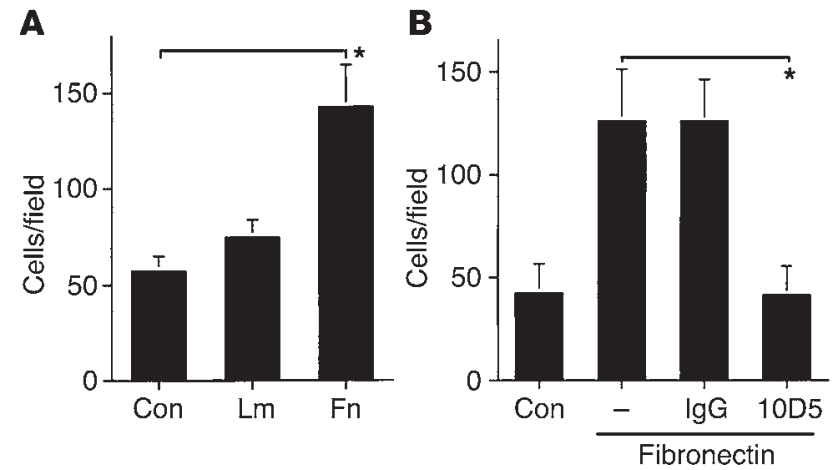

Figure 3

Increased $\alpha \mathrm{v} \beta 6$ expression promotes migration on fibronectin. (A) Chemotactic migration assay of post-EMT LIM 1863 cells for 3 days on untreated control Transwells (Con), or Transwells coated with laminin $(\mathrm{Lm})$ or fibronectin $(\mathrm{Fn})$. Data are expressed as means and SDs of 8 individual fields randomly selected from each well, with each experiment performed in triplicate. ${ }^{*} P<0.05$. (B) LIM 1863 cells were subjected to the chemotaxis assay as described, on untreated (Con) or fibronectin-coated Transwells as indicated. Chemotaxis on fibronectin was performed in the absence $(-)$ or presence of function-blocking anti- $\beta 6$ monoclonal (10D5) or isotype-matched control (IgG) antibody, at a concentration of $100 \mu \mathrm{g} / \mathrm{ml}$. The anti- $\beta 6$ antibody reduced chemotaxis back to the levels of the uncoated migration control for these cells. ${ }^{\star} P<0.05$.

indicate that upregulation of the $\beta 6$ integrin subunit mRNA and a consequent increase in $\alpha v \beta 6$ expression occur during the EMT of LIM 1863 colon carcinoma cells.

Transcriptional control of $\beta 6$ integrin by the Ets-1 transcription factor. Although transcriptional regulation of the $\beta 6$ integrin has not been studied, we reported recently that the expression and activity of the Ets-1 transcription factor are induced during the EMT (12). This proto-oncogene is a member of a family of transcription factors that regulate genes involved in development, cellular differentiation, and cell proliferation (13). Interestingly, we identified 4 potential consensus Ets-binding sites in the first $1 \mathrm{~kb}$ of the human $\beta 6$ promoter (numbered 1-4, with 1 being most proximal to the transcription start site; Figure 2A). To determine whether Ets- 1 is capable of transactivating the $\beta 6$ promoter, we performed luciferase assays with a number of Ets family members (Figure 2B). Ets-1 displayed a robust activation of the $\beta 6$ promoter (greater than 2.5 -fold), whereas none of the other family members tested showed any inducing effect. The closely related factor Ets-2, as well as Mef and NERF2, failed to activate the promoter to a greater degree than the PCI control did, while the others tested repressed activity (Figure 2B). Because all these family members bind the same core recognition sequence, this finding strongly argues for selectivity of Ets- 1 in promoting $\beta 6$ integrin transcription. To assess the relative contributions of each of these 4 potential regulatory sites, we performed gel shift assays. Ets-1 bound strongly to site 1 , and no binding was evident for any of the other 3 more distal sequences (Figure 2C). To substantiate this result, we performed site-directed mutagenesis of the core recognition sequence GGAA to TTAA at this first site. When increasing doses of wild-type Ets- 1 were titrated into the transcription assay, a clear dose-response effect of $\beta 6$ promoter activity was observed (Figure 2D). In contrast, mutagenesis of site 1 completely abrogated any luciferase activity, independent of dose, thus confirming the specificity and importance of this Ets-1-binding site for regulating $\beta 6$ transcription.

Elevated $\alpha \nu \beta 6$ expression promotes tumorigenic functions following $E M T$. To ascribe a functional role for increased $\alpha v \beta 6$ expression during the EMT, we focused initially on its classical role as a fibronectin receptor (14). Although LIM 1863 organoids are nonmotile (11), they are capable of undergoing chemotaxis following EMT (10). Indeed, using Transwells (Corning Inc.) coated with specific matrix proteins, we observed that post-EMT cells were significantly more chemotactic when the Transwells were coated with fibronectin than when they were not coated or were coated with laminin-1 (Figure 3A). This migration is mediated by $\alpha v \beta 6$ and is not attributable to an alternative compensatory response of the post-EMT phenotype, since it was inhibited by a functionblocking $\beta 6$ antibody (Figure 3B). These data indicate that induction of $\alpha v \beta 6$-mediated migration on fibronectin is a consequence of the EMT of LIM 1863 cells.

TGF- $\beta$ is a central mediator of the EMT process $(5,6,15)$, and it is the exogenous application of this cytokine that drives the EMT in the LIM 1863 spheroid model (10). Unexpectedly, we discovered that LIM 1863 cells undergoing EMT exhibit autocrine expression and secretion of TGF- $\beta$ (Figure 4A). Organoids or cytokine-treated cells were seeded for 7 hours in RPMI medium supplemented with $5 \%$ FCS, washed 3 times with serum-free medium to remove the endogenous TGF- $\beta$ present in serum, and then cultured for an additional 17 hours. Serum-free media contained no TGF- $\beta$, whereas the final of the 3 washes contained background levels derived from the FCS (Figure 4A). TGF- $\beta$ production by intact organoids was negligible compared with the residual TGF- $\beta$ levels of the controls. In contrast, post-EMT cells secreted high levels of TGF- $\beta$, confirming the existence of autonomous TGF- $\beta$ production in these cells.

TGF- $\beta$ family members are secreted as latent complexes that require activation in order to bind their receptors, and an essential role for $\alpha v \beta 6$ to bind and activate latent TGF- $\beta$ has emerged (16).
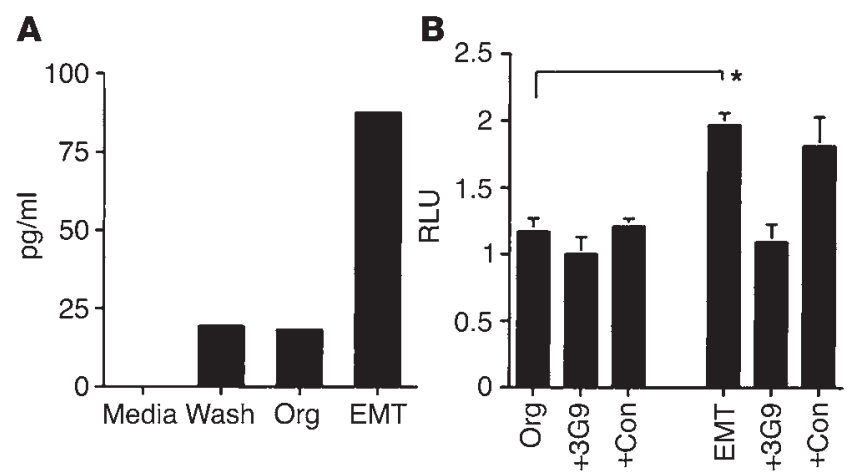

\section{Figure 4}

Autocrine TGF- $\beta$ production and activation by $\alpha \mathrm{v} \beta 6$ in EMT cells. (A) ELISA was performed to measure secreted TGF- $\beta$ levels in serumfree media (Media), media after washing (Wash), or overnight culture media from organoid or EMT cultures of LIM 1863 cells (Org, EMT). (B) Reporter and LIM 1863 cells were cultured 16-20 hours and lysed for measurement of luciferase activity. Results are for organoids (Org) and EMT cells (EMT). Addition of function-blocking anti- $\beta 6$ monoclonal (3G9, $30 \mu \mathrm{g} / \mathrm{ml}$ ) or control antibody (Con) is shown at the bottom. Relative luciferase activity (RLU) is the measured activity divided by the activity of the coculture of organoid (control) cells in the presence of function-blocking antibody. ${ }^{*} P<0.001$. 

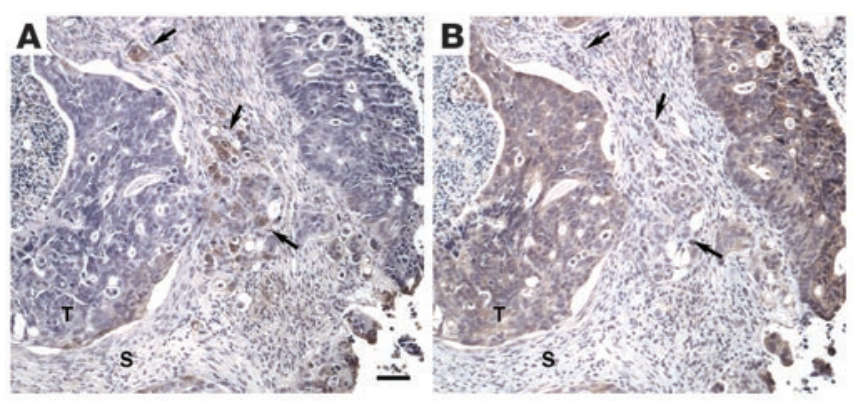

\section{Figure 5}

The integrin $\alpha v \beta 6$ is a marker of EMT in vivo. Integrin $\beta 6$ (A) or E-cadherin (B) immunostaining of sequential tumor sections derived from LIM 1863 xenografts. E-cadherin expression is prominent in the tumor tissue $(T)$ and absent from the stroma (S). $\beta 6$ Immunoreactivity is relatively weak in the tumor masses, but it is strongly upregulated in tumor cells invading the stroma, which are also negative for E-cadherin expression (arrows). Scale bar: $50 \mu \mathrm{m}$.

Therefore we assessed whether the $\alpha v \beta 6$ induced during the EMT is capable of activating TGF- $\beta$, by coculturing intact organoids or post-EMT cells with mink lung epithelial reporter cells stably expressing a portion of the plasminogen activator inhibitor 1 promoter, as described previously $(16,17)$. Coculture with the post-EMT cells caused a significant increase in luciferase levels compared with coculture with control (organoid) cells (Figure 4B). Significantly, this increase was abolished by a function-blocking $\mathrm{mAb}$ against $\beta 6$ (3G9), which indicates that this effect is completely attributable to the increased expression of $\alpha v \beta 6$ receptor on these cells. Taken together, these results show that LIM 1863 cells induced to undergo an EMT not only produce an autocrine supply of TGF but also have the capacity to process and activate the latent form of the cytokine using the $\alpha v \beta 6$ integrin.

$E M T$ and $\alpha \nu \beta 6$ expression in vivo. Having found that upregulation of $\alpha v \beta 6$ is a consequence of the EMT process in vitro, we sought to establish a relationship among EMT, $\beta 6$ expression, and tumor progression in vivo. To achieve this, LIM 1863 xenograft tumors were grown s.c. in nude mice, before immunohistochemical analysis of relevant markers. As expected, these organoid-derived tumors stained only weakly for $\beta 6$ (Figure 5A). However, there was a dramatic induction of $\beta 6$ expression in localized regions of the tumors, such as is shown in Figure 5A, and in particular on tumor cells that were invading the stroma. Thus, tumor cells that were migrating out from an invasive front displayed a clear increase in receptor expression. Significantly, when a sequential tumor section was stained for E-cadherin, the in situ tumor cells were strongly positive, whereas the invading cells had completely lost expression of this molecule (Figure 5B). Based on our model, we conclude that the invading cells had undergone an EMT in vivo (most likely the result of stromal factors), resulting in loss of the epithelial marker E-cadherin and a concomitant upregulation of $\beta 6$. These morphological data not only support our hypothesis that elevated $\beta 6$ expression facilitates the invasion and dissemination of colon carcinoma cells but also provide direct evidence that the EMT correlates with disease progression.

High $\alpha \nu \beta 6$ expression in buman colon carcinoma is a prognostic indicator of poor survival. Based on our EMT data, we predicted that the frequency of $\alpha v \beta 6$ expression in human disease is skewed toward late-stage, more aggressive tumors, rather than adenomas and in situ carcinomas. To examine this postulate, we performed immunohistochemistry on tissue arrays of malignant colorectal tumors with known clinical outcome (Figure 6). Table 1 summarizes the patient characteristics for 488 successfully stained primary carcinoma samples, of which 181 (37\%) were positive for $\beta 6$ expression. The median age of patients was 68 years, with a range from 23 to 94. For subsequent analysis, the staining intensity of $\beta 6$ was scored as 0 , 1 , or 2 . Of the 488 samples, 307 (63\%) were negative (score 0), 95 (19\%) exhibited low expression (score 1), and 86 $(18 \%)$ exhibited high expression (score 2 ) (Table 1).

Normal colonic mucosa did not exhibit any staining for $\beta 6$ (Figure 6A). A tumor sample negative for $\beta 6$ expression is shown in Figure $6 \mathrm{~B}$, and, in contrast, an example of strong immunoreactivity of $\beta 6$ expression (score 2 ) is shown in Figure $6 \mathrm{C}$. Consistent with the hypothesis that $\alpha v \beta 6$ expression occurs as a function of tumor progression, high $\beta 6$ expression was detected in the more poorly differentiated infiltrating tumor cells invading through desmoplastic stroma (Figure 6D). Heterogeneous distribution patterns were frequently observed (e.g., Figure 6E) that displayed an intense upregulation and preferential localization of the integrin at the edges of infiltrating tumor islands. A sequential serial section of this particular tumor was used as a negative control (Figure 6F).

We then examined whether there was an association between $\alpha v \beta 6$ expression and patient survival. We analyzed the data by considering only disease-related death as an event, censoring deaths unrelated to disease and the patients who were alive when they were last seen. To determine differences in the cancer survival among patients who differed in $\alpha v \beta 6$ expression (scores $0-2$ ), the log-rank test was performed, and the corresponding Kaplan-Meier plot is shown in Figure 7. The log-rank test indicated there was no significant survival difference between patients with score 0 and patients with score $1(P=0.99)$. There was, however, a significant difference

\section{Figure 6}

Expression of $\alpha v \beta 6$ in malignant human colon carcinoma. (A-E) Representative $\beta 6$ immunostaining of normal human colon $(\mathbf{A})$ or malignant colon carcinoma tissue (B-E). A negative (B) and a positive (C) tumor sample are shown. $\beta 6$ Immunostaining is shown for a separate carcinoma sample in $\mathbf{D}$, with tumor cells infiltrating the stroma showing high expression of the receptor. Heterogeneous receptor expression in another primary tumor, with intense upregulation and preferential localization to tumor islets, is illustrated in E. (F) The corresponding negative control for the tumor shown in E. Scale bars: $100 \mu \mathrm{m}(\mathbf{A}-\mathbf{C}, \mathbf{E}$, and $\mathbf{F})$ and $50 \mu \mathrm{m}$ (D).

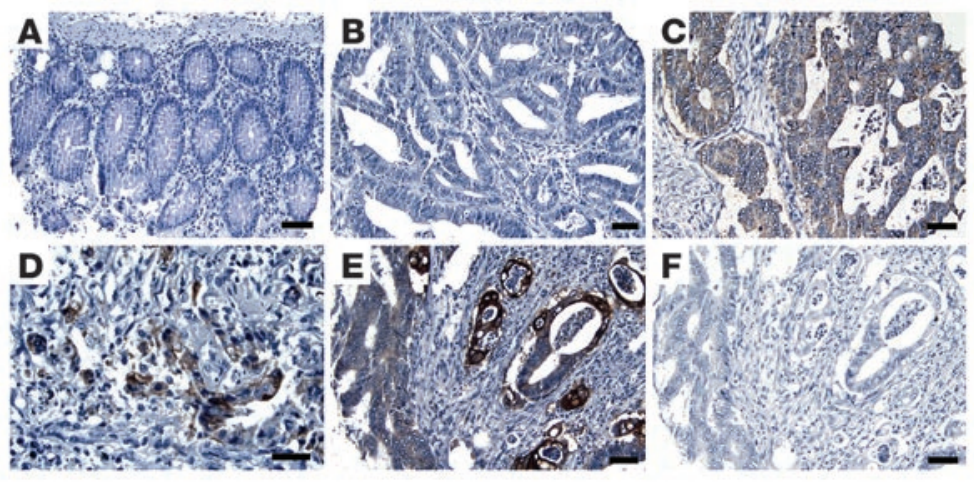


Table 1

Patient characteristics

\begin{tabular}{lcc} 
Characteristics & No. of patients & Percent \\
Sex & & \\
Unknown & 17 & 3.5 \\
Male & 211 & 43.2 \\
Female & 260 & 53.3 \\
Lymph node status & & \\
Unknown & 75 & 15.4 \\
N0 & 232 & 47.5 \\
N1 & 112 & 23.0 \\
N2 & 66 & 13.5 \\
NX & 3 & 0.6 \\
Stage & & \\
Unknown & 42 & 8.6 \\
1 & 102 & 20.9 \\
2 & 124 & 25.4 \\
3 & 176 & 36.1 \\
4 & 44 & 9 \\
Tumor type & & \\
Unknown & 18 & 3.6 \\
Colon & 360 & 73.8 \\
Other digestive organ & 1 & 0.2 \\
Rectum & 108 & 22.1 \\
Small intestine & 1 & 0.2 \\
Score ( $\beta 6$ expression) & & \\
0 & 307 & 19.5 \\
1 & 95 & \\
2 & 86 & \\
\hline
\end{tabular}

between patients with score 0 and patients with score $2(P=0.048)$, and between patients with score 0 or 1 and patients with score 2 $(P=0.04)$. Further, the Kaplan-Meier plot showed that patients with score 0 or 1 (i.e., either no expression or low expression of $\beta 6$ ) had longer survival times than patients with score 2 (high expression of $\beta 6$ ). For this reason, we combined the patients with a score of 0 or 1 in the data set and compared their survival with that of the patients with score 2 . The survival estimates showed a striking difference in median survival between the 2 groups: the group with a score of 0 or 1 averaged 16.5 years, whereas that with a score of 2 averaged less than 5 years and showed a $15 \%$ lower 5 -year survival rate (Table 2). From a biological perspective, we conclude that elevated expression of $\alpha v \beta 6$ is a prognostic indicator of poor survival for patients with colorectal carcinoma. This key finding substantiates the data predicted by the spheroid model.

The log-rank results suggested a significant interaction between the effects of score and stage, and it was possible that the survival effects of $\beta 6$ simply correlated with the degree of invasion. For this reason, we separated the expression data by stage, either early (stages 1 and 2) or late (stages 3 and 4) (Table 2). For patients with stage 3 or 4 tumors, high expression of $\beta 6$ resulted in a minor (4-5\%), not significant, reduction in both 5 - and 10-year survival rates. In stark contrast, elevated $\beta 6$ expression had a much more significant impact on the survival of those patients with early-stage tumors $(P=0.0015)$, highlighted by a greater-than-28\% reduction in 5-year survival compared with that of patients with no expression or low expression.
Importantly, these data define $\beta 6$ expression as an independent prognostic variable for colorectal cancer that is predictive of outcome in early-stage disease.

Based on the above findings, the proportional hazard ratio model was used to obtain more precise estimates of the effects of $\beta 6$ expression on survival by adjusting for the effects of age, sex, stage, and tumor type. Table 3 summarizes the $\chi^{2} P$ values, the hazard ratios, and the $95 \%$ confidence interval of hazard ratio, for all variables. Again, when score 0 and 1 patients (no expression or low expression) were compared with score 2 patients (high expression), the $P$ value of 0.007 revealed a significant difference in survival. As expected, the standard prognostic indicators of tumor stage and age at detection each showed significant effects on survival. Significantly, the hazard ratio of 1.6 for elevated $\beta 6$ expression indicated that the hazard of dying was $60 \%$ higher in patients with score 2 than in patients with score 0 or 1 . This result confirmed the findings of the log-rank test in identifying $\beta 6$ expression as an independent variable and, importantly, suggested that high $\beta 6$ expression in colon tumors represented a significant risk factor for patient survival.

Expression of $\alpha v \beta 6$ in human colon cancer metastases. In Figure 8 we provide evidence of $\alpha v \beta 6$ expression in human colon carcinoma metastases as assessed by immunohistochemistry on lymph nodes and liver specimens obtained from patients with colorectal cancer. Infiltrating colon carcinoma cells were easily discernible by $\mathrm{H} \& \mathrm{E}$ staining of a section of lymph node from a patient with invasive colon cancer (Figure 8A). $\beta 6$ Staining of the same tissue showed strong immunoreactivity in the tumor cells, while the lymphatic cells of the organ were negative, as expected (Figure 8B). To define receptor expression in more distal organs, we stained metastatic lesions in randomly selected liver biopsies (Figure 8, C and D). The colonic metastases were readily distinguished from the hepatic tissue by $\mathrm{H} \& \mathrm{E}$ staining (Figure 8C), and these tumor cells again displayed strong $\beta 6$ staining (Figure 8D; 3 of 4 cases). Negative-control tissue staining showed no immunoreactivity, as expected (data not shown).
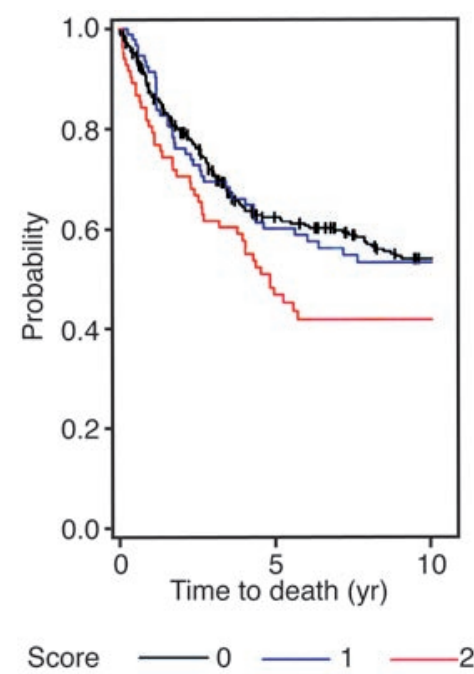

\section{Figure 7}

Kaplan-Meier survival analysis using a log-rank test. The samples were grouped according to $\beta 6$ expression level (score 0 , negative; score 1, low expression; score 2, high expression) and analyzed using the log-rank test for overall survival. 


\section{Table 2}

Survival estimates for disease-related deaths

$\begin{array}{lccc}\text { Group } & \text { Median survival (yr) and 95\% Cl } & \mathbf{5 - Y e a r ~ s u r v i v a l ~ r a t e ~ ( \% ) ~ a n d ~ 9 5 \% ~ C l ~} & \mathbf{1 0 - Y e a r ~ s u r v i v a l ~ r a t e ~ ( \% ) ~ a n d ~ 9 5 \% ~ C l ~} \\ \text { Score 0-1 } & 16.5(8.2, \text { not estimable) } & 61.7(56.6,66.7) & 53.7(48.3,59.0) \\ \text { Score 2 } & 4.8(3.2, \text { not estimable) } & 46.7(35.5,57.8) & 41.7(30.3,52.9) \\ \text { Stage 1-2 and score 0-1 } & \text { Not reached } & 77.5(71,84) & 69.6(62.2,77.1) \\ \text { Stage 1-2 and score 2 } & 5.0(3.2, \text { not estimable) } & 49.3(35,63.6) & 46.4(31.9,61) \\ \text { Stage 3-4 and score 0-1 } & 3.8(2.9,6.9) & 45.7(38.4,53.1) & 37.8(30.4,45.1) \\ \text { Stage 3-4 and score 2 } & 4.4(1.8, \text { not estimable) } & 41.7(22.8,60.6) & 32.4(13.9,51)\end{array}$

Log-rank test suggested significant survival difference between groups: stage $1-2$ vs. $3-4(P<0.0001)$, score $0-1$ vs. $2(P=0.04)$, and score $0-1$ vs. 2 among patients at stage $1-2(P<0.0015)$. There was no significant difference between score $0-1$ and score 2 among patients at stage $3-4(P=0.65)$. $\mathrm{Cl}$, confidence interval.

The demonstration of $\alpha v \beta 6$ expression in distal metastases supports our conclusion that $\alpha v \beta 6$ expression in colorectal cancer correlates with disease progression and poorer clinical outcome.

\section{Discussion}

The progression to invasive and metastatic carcinoma involves profound alterations in epithelial structure and function. We show here that the EMT of colon carcinoma is coincident with an increase in $\alpha v \beta 6$ integrin expression, and the culmination of our observations is that high expression of $\beta 6$ in primary colon carcinomas is a prognostic marker for aggressive disease and patient mortality. This integrin is expressed primarily during development, and high $\alpha v \beta 6$ expression in adults is limited only to a few epithelial tissues $(18,19)$. However, $\alpha v \beta 6$ can be reexpressed in parallel with specific morphogenetic events such as inflammation and wound healing (19). Wound healing, in particular, is believed to involve EMT processes, and the induction of $\alpha v \beta 6$ implies a central role for the receptor (19-21). In addition, several studies have shown neo-expression of $\alpha v \beta 6$ in oral squamous cell carcinomas $(19,22,23)$, although a link between expression and patient outcome has not been established. Nonetheless, these studies are consistent with our observation that $\alpha v \beta 6$ expression is induced as a consequence of EMT. Interestingly, studies on the phenotypic changes induced by the EMT have traditionally focused on the transcriptional repression of epithelial markers that maintain tissue polarity, such as E-cadherin, and the functional consequences of induced mesenchymal markers. Our discovery that an epithelial integrin is dynamically regulated during the EMT of colon carcinoma, and specifically one that is upregulated during development, is consistent with the long-standing hypothesis that colorectal carcinogenesis more accurately reflects a dedifferentiation of epithelia to a more embryonic phenotype.

The induction of $\alpha v \beta 6$ expression that we observed during the EMT is dependent on the transcriptional upregulation of the $\beta 6$ integrin subunit. The transcriptional regulation of $\beta 6$ had not been investigated previously to our knowledge, and it now appears that Ets-1 may be a key transcription factor that influences EMT. In fact, we have recently reported that Ets-1 expression is upregulated as a consequence of the EMT, and that it is responsible for the induction of the VEGF receptor Flt-1 (12). A functional role for Ets- 1 in $\beta 6$ regulation was evidenced by its ability to bind and transactivate the human $\beta 6$ promoter. Our screening identified 4 putative consensus sites within the first kilobase of the promoter sequence, but only the first site appeared to be of importance. Ets-1 bound preferentially to this site in gel shift assays, and, most significantly, site-directed mutagenesis of this region completely abrogated transactivation activity. Since Ets-1 typically exerts its effects in cooperation with other transcriptional factors and cofactors, we are currently seeking to identify other regulators of $\beta 6$ transcription. The role, if any, of the other 3 consensus sites also awaits further characterization. Although they appear dispensable for $\beta 6$ regulation during the EMT process, the possibility exists that alternate triggers, or tissue-specific factors, might act to influence $\beta 6$ gene regulation via these sites.

We define 2 key functions of $\alpha v \beta 6$ that can be linked to the biology of aggressive colon carcinoma: activation of autocrine TGF- $\beta$, and promotion of migration on fibronectin matrices. Firstly, TGF- $\beta$ has been proposed to act as a cell-autonomous promoter of late-stage human tumor development because of its ability to regulate EMT (5). In this context, the autocrine TGF- $\beta$ production we observed would provide the means to stabilize and sustain the EMT by continuous TGF- $\beta$ receptor signaling in a cell-autonomous fashion. However, it is the ability of $\alpha v \beta 6$ to activate autocrine TGF- $\beta$ in post-EMT cells that is most novel with respect to colon carcinoma progression. The recognition that this integrin can bind and activate latent TGF- $\beta$ (16) identified a likely role for this receptor in contributing to the onset of TGF- $\beta$-mediated diseases, such as inflammation and fibrosis $(24,25)$. From our perspective, induced expression of $\alpha v \beta 6$ in colon carcinoma would provide a mechanism to locally regulate TGF- $\beta$ function in vivo, to provide a feedback loop to perpetuate the EMT process, and, in turn, to create a tumor microenvironment more amenable to progression. Indeed, the morphological analysis of the xenografts (Figure 5) provides in vivo evidence for this concept.

\section{Table 3}

Multivariate analysis for disease-related deaths (Cox regression model)

$\begin{array}{lccc}\text { Variable } & \boldsymbol{P} \text { value } & \text { Hazard ratio } & \mathbf{9 5 \%} \mathbf{~ C l} \\ \text { Group (0-1 vs. 2) } & 0.007 & 1.60 & (1.14,2.26) \\ \text { Age } & <0.0001 & 1.03 & (1.02,1.05) \\ \text { Sex } & 0.03 & 0.73 & (0.56,0.97) \\ \text { Stage (1-2 vs. 3-4) } & <0.0001 & 2.64 & (1.96,3.56) \\ \text { Tumor type } & 0.03 & 0.7 & (0.51,0.97) \\ \quad \text { colon vs. rectum/other) } & & & \end{array}$




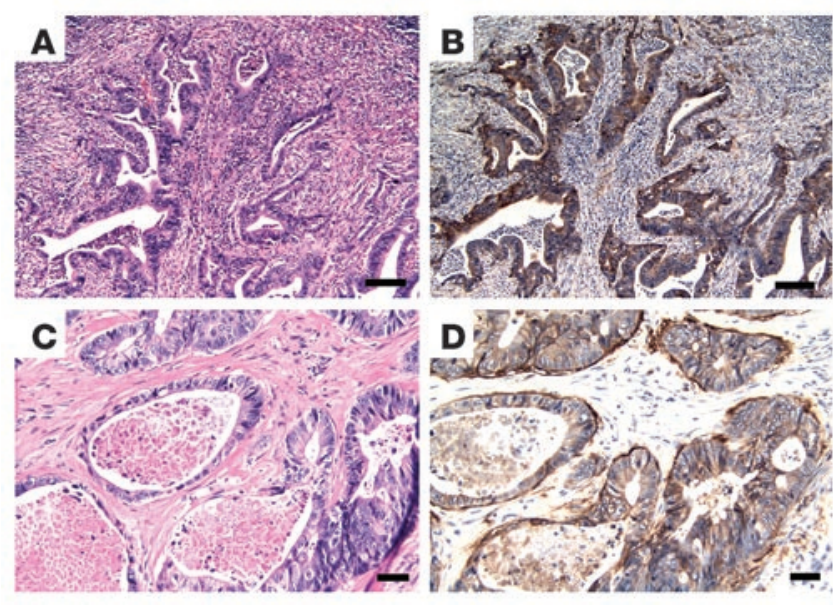

Figure 8

In vivo $\alpha v \beta 6$ protein expression in human colorectal metastases. Representative $\mathrm{H} \& \mathrm{E}$ (A and $\mathbf{C}$ ) or $\beta 6$ immunohistochemistry (B and $\mathbf{D})$ in lymph node ( $\mathbf{A}$ and $\mathbf{B}$ ) or liver tissue ( $\mathbf{C}$ and $\mathbf{D}$ ) containing human colorectal metastases. $\beta 6$ Immunoreactivity in both samples is restricted to the metastasized tumor cells. Scale bars: $100 \mu \mathrm{m}$ (A and B) and $50 \mu \mathrm{m}$ (C and D).

Secondly, having a specific fibronectin receptor elicited as a consequence of the EMT also has selective advantages for tumor cells. The colonic epithelium resides upon a basement membrane, composed primarily of laminins, collagen IV, and proteoglycans, which provides an interface with the lamina propria beneath (26). The structural composition of this zone differs in its high fibrillar collagen content, and also in enrichment with fibronectin and tenascin, both ligands for $\alpha v \beta 6(14,27)$. Thus, an enhanced migratory capacity on fibronectin would facilitate the escape and dissemination of invading colon carcinoma cells into the lamina propria. Moreover, $\alpha v \beta 6$ expression and fibronectin recognition may also be important for colonization of the liver, the primary site for metastases derived from colorectal tumors. In addition to the anatomical consideration that portal blood flow from the colon goes directly to this organ, our data suggest that there may be an additional dimension to colonization that specifically involves $\alpha v \beta 6$. Since there is no basement membrane under the endothelium of liver microvessels as in other tissues, it has been suggested that adhesion to fibronectin may be of central importance for liver metastasis, because it is abundant on the surface of hepatocytes (28). We propose, therefore, that an increased propensity to adhere to fibronectin would promote colon carcinoma metastasis to the liver by aiding extravasation.

The key conclusion of this study relates to the expression of $\alpha v \beta 6$ in human colorectal cancer. We identified this receptor as a marker of aggressive carcinoma, in part based on its high frequency of expression (almost 40\%) in malignant tumors. Additional compelling evidence for the involvement of $\alpha v \beta 6$ in tumor progression is provided by our demonstration of $\beta 6$ expression in both lymphatic and hepatic metastases derived from human colorectal carcinomas. However, the result of greatest consequence is that analysis of the clinical data revealed that elevated $\beta 6$ expression, rather than the simple presence or absence of the receptor, is linked to significantly reduced survival times. Even more striking was the discovery that its value as a prognostic marker is more significant for early-stage tumors. It is likely that the effects of $\beta 6$ expression on late-stage tumors are masked by additional genetic and epigenetic alterations. However, based on our results, it is tempting to speculate that the EMT process can contribute directly to tumor progression and development of metastatic disease, and that $\alpha v \beta 6$ represents a novel marker for this event. Moreover, the hazard ratio analysis confirmed that this receptor is a stand-alone variable, similar to those of stage and age at diagnosis, for colorectal tumors, and indicated that high expression of this integrin is an independent and important risk factor for patient survival. Overall, these findings establish $\alpha v \beta 6$ as a novel prognostic indicator for human colon cancer and validate our EMT model as a valuable tool for the identification of clinically relevant markers.

Finally, a potentially important consequence of our work is that $\alpha v \beta 6$ may be an attractive therapeutic candidate for colon cancer and, in particular, that it may be possible to selectively target invading and metastasizing cells. Furthermore, because elevated $\beta 6$ expression is predictive of outcome in early-stage disease, it may also be a feasible target for earlier intervention and treatment. Targeting integrin function has emerged as a legitimate therapeutic strategy for several disease types. In this regard, the current development and characterization of function-blocking anti- $\beta 6$ antibodies (29) offer the promise of new reagents and novel treatments not only for diseases such as fibrosis but also, in light of the findings presented here, for aggressive colorectal cancer.

\section{Methods}

Cell culture and EMT induction. LIM 1863 cells $(11,30)$ were grown as organoids in RPMI 1640 (GIBCO; Invitrogen Corp.) supplemented with $5 \%$ FCS. To induce the EMT, LIM 1863 cells were seeded in 24-well plates with a combination of TNF- $\alpha(10 \mathrm{ng} / \mathrm{ml})$ and TGF- $\beta 1(2 \mathrm{ng} / \mathrm{ml})$ for 24 hours (10). Morphological changes in the organoids were assessed by phase-contrast microscopy. The cell line HEK293 (human embryonic kidney) was grown in DMEM supplemented with 10\% FCS and antibiotics (penicillin and streptomycin) (GIBCO; Invitrogen Corp.). Mink lung epithelial cells stably transfected with a plasmid containing the luciferase cDNA downstream of a TGF- $\beta$-sensitive portion of the plasminogen activator inhibitor 1 promoter were grown in DMEM supplemented with $4.5 \mathrm{~g} / \mathrm{l}$ glucose and $10 \% \mathrm{FCS}$.

Antibodies. The murine mAb directed against $\alpha \mathrm{v}$ integrin, 13C2, was a kind gift from M. Horton (Imperial Cancer Research Fund, London, United Kingdom). The anti- $\beta 6 \mathrm{mAbs} 3 \mathrm{G} 9$ and $2 \mathrm{G} 2$ were obtained from S. Violette (Biogen Idec, Cambridge, Massachusetts, USA), and the specificity of these antibodies has recently been reported (29). The anti- $\beta 6$ antibody CS $\beta 6$ and function-blocking monoclonal 10D5 were purified in our laboratories, and control mouse Igs were purchased from SigmaAldrich. The polyclonal E-cadherin antibody (H-108) was purchased from Santa Cruz Biotechnology Inc.

Real-time quantitative PCR. Real-time quantitative PCR was performed on RNA samples that were first reverse-transcribed and then treated with DNase. The primers and probes (listed below) were designed using Primer Express software version 1.0 (Applied Biosystems), based on mRNA sequences obtained from the National Center for Biotechnology Information database (31). All reactions were performed in an ABI PRISM 7700 Sequence Detection System (PerkinElmer Applied Biosystems). Reactions were carried out in triplicate in a $50-\mu l$ reaction volume containing $25 \mu \mathrm{l}$ of $2 \times$ TaqMan PCR Master Mix, a 50-nM concentration of each forward and reverse primer, a 100-nM concentration of duallabeled probe, and $1 \mu \mathrm{g}$ of total cDNA. Conditions for all PCR reactions were 2 minutes at $50^{\circ} \mathrm{C}$ and 10 minutes at $95^{\circ} \mathrm{C}$, followed by 40 cycles of $95^{\circ} \mathrm{C}$ for 10 seconds and $60^{\circ} \mathrm{C}$ for 1 minute. Normalization to actin 
(housekeeping gene) was performed for each sample. Cycle threshold values were exported into an Excel (Microsoft Corp.) worksheet for calculation of fold changes according to the $\Delta \Delta \mathrm{CT}$ method. The primers and dual-labeled probe (TET/TAMRA) were as follows: $\beta 6$ integrin sense, 5'-CTACCTGTGGTGACCCCTGTAAC-3'; $\beta 6$ integrin antisense, $5^{\prime}$ GCTTGGCCAGCTGCTGAC-3'; $\beta 6$ integrin probe, 5'/5TET/CTAAACGGAGCTGCATTGAGTGCCACC/36-TAM/3'.

Expression vector and luciferase reporter gene constructs. An 1,134-bp fragment corresponding to nucleotides -926 to +208 of the human ITG $\beta 6$ promoter was cloned from human genomic DNA (BD Biosciences Clontech) by PCR and subcloned into the PGL2 luciferase reporter vector (Promega Corp.). The fragment was inserted into the NheI-XhoI site upstream of the luciferase gene of the pGL2 vector.

DNA transfection assays. Cotransfections of $2.5 \times 10^{5} \mathrm{HEK} 293$ cells were carried out with varying amounts of reporter gene construct DNA and expression vector DNA using $4 \mu \mathrm{l}$ LipofectAMINE (Invitrogen Corp.) as previously described (32). The cells were harvested 16 hours after transfection and assayed for luciferase activity. Transfections for every construct were performed independently and in duplicate. Cotransfection of a second plasmid for determination of transfection efficiency was omitted, because potential artifacts with this technique have been reported and because many commonly used viral promoters contain potential binding sites for Ets factors (33).

Site-directed mutagenesis. Site-directed mutagenesis of the ITG $\beta 6$ was performed using the QuikChange Site-Directed Mutagenesis Kit (Stratagene) according to the manufacturer's recommendations. In brief, PCR primers encoding the ITG $\beta 6$ promoter Ets site, -66 to -63 , and flanking sequences were used, with TTAA substituted for GGAA (mutation underlined): 5'AACATGATTTACTTAATTGTTCTCCTTATAAGGAAGGCAGG-3' and 3'-CCTGCCTTCCTTATAAGGAGAACAATTAAGTAAATCATGTT-5'. PCR was performed with PfuTurbo polymerase (Stratagene) using the wild-type ITG $\beta 6$ promoter luciferase reporter construct as a template. The PCR reaction was digested with $D p n I$, and the undigested plasmids were transformed into DH5 $\alpha$ bacteria. Individual plasmid preparations were sequenced to verify incorporation of the Ets site mutation.

Electrophoretic mobility shift assay. Electrophoretic mobility shift assays were performed as described previously (34). In brief, $2 \mu$ l of in vitro translation product and 0.1-0.2 ng [ $\left.{ }^{32} \mathrm{P}\right] \mathrm{dATP}-$ labeled double-stranded oligonucleotide probes $(5,000-20,000 \mathrm{cpm})$ were run on $4 \%$ polyacrylamide gels containing $0.5 \times$ Tris-Borate-EDTA buffer. Oligonucleotides used as probes were as follows (Ets consensus binding site underlined): human ITG $\beta 6$ promoter site -73 to $-51,5^{\prime}$-AGAACAAGGAAGTAAATCATGTT-3' and $3^{\prime}$-AACATGATTTACTTCCTTGTTCT-5'.

Immunoprecipitation and immunoblot analysis. To assess $\beta 6$ integrin protein levels, cells were extracted in a Triton X lysis buffer (1\% Triton X-100, 50 $\mathrm{mM}$ Tris, $150 \mathrm{mM} \mathrm{NaCl}$ ) containing protease inhibitors (pepstatin, PMSF, aprotinin, and leupeptin) for 1 hour. Extracts were clarified by centrifugation. For immunoprecipitations, the lysates were precleared twice with anti-mouse IgG-agarose (Sigma-Aldrich). Immunoprecipitations were carried out indirectly using the monoclonal $13 \mathrm{C} 2$ or isotype-matched control IgG and captured with anti-mouse IgG-agarose. Extracts and precipitates were analyzed by SDS-PAGE, and proteins were transferred to nitrocellulose by electrophoresis. Residual protein sites were blocked in Tween/Tris-buffered saline (TBST) containing 5\% skim milk. The filters were incubated with an $\mathrm{mAb}$ directed against $\beta 6(2 \mathrm{G} 2)$ in TBST plus $2.5 \%$ skim milk for 1 hour and developed using ECL.

Xenograft studies. LIM 1863 organoids (approximately $3 \times 10^{6}$ cells) were inoculated s.c. into the flanks of female nude mice. The animals were 6-7 weeks of age at the time of tumor implantation. Tumor xenografts were harvested 16 weeks later, and formalin-fixed, paraffin-embedded sections were used for immunohistochemistry. All animal studies were approved by the Institutional Animal Care and Use Committee (IACUC) of Beth Israel Deaconess Medical Center.

Flow cytometry. Cells were incubated with either the anti- $\beta 6 \mathrm{mAb} 3 \mathrm{G} 9$ or an isotype-matched control IgG (10 $\mu \mathrm{g} / \mathrm{ml}$ in PBS) for 30 minutes. After washing, cells were resuspended in FITC-conjugated secondary antibody for 30 minutes in the dark, prior to FACScan analysis (BD Biosciences).

Immunohistochemistry. Immunohistochemistry was performed using the avidin-biotin complex protocol on paraffin-embedded tissue. Tissue microarray slides were generated as previously described (35). Briefly, formalin-fixed, paraffin-embedded tissue blocks containing colon carcinoma specimens were retrieved from the archives of the Yale University Department of Pathology (New Haven, Connecticut, USA) under Human Investigative Committee (HIC) protocol no. 8219. To generate the microarray, areas of invasive carcinoma were selected for coring and placement into a recipient master block using a Tissue Microarrayer (Beecher Instruments Inc.). Cores measured $0.6 \mathrm{~mm}$ in greatest dimension and were spaced $0.8 \mathrm{~mm}$ apart. The tissue microarray was sliced into $5-\mu \mathrm{m}$ sections and adhered to slides by an adhesive tape-transfer system (Instrumedics Inc.) and UV cross-linking. For the metastasis staining, 4 randomly selected cases of liver metastasis and 2 samples of lymph node containing colorectal metastases were selected for study from the Beth Israel Deaconess Medical Center, Department of Pathology (tissue procurement was approved by the Institutional Review Board). For immunohistochemistry, the slides were deparaffinized, and endogenous peroxidase activity was blocked by incubation in a hydrogen peroxide/methanol buffer. Antigen retrieval was then performed by incubation of the slides in a pepsin solution (Zymed Laboratories Inc.) at $37^{\circ} \mathrm{C}$. Overnight incubation at $4^{\circ} \mathrm{C}$ with primary antibody (2G2, $2 \mu \mathrm{g} / \mathrm{ml}$; E-cadherin, $0.04 \mu \mathrm{g} / \mathrm{ml}$ ) in $0.1 \%$ BSA was preceded by blocking with $1 \times$ casein solution (Vector Laboratories Inc.). Negative controls were performed by substitution of the primary antibody with PBS. The following day, biotinylated anti-mouse IgG (1:200; DakoCytomation) was applied to the slides, which were subsequently treated using a Vectastain ABC kit (Vector Laboratories Inc.). Slides were incubated for 10 minutes with a peroxidase solution (DAKO EnVision+ System, Peroxidase [DAB]; DakoCytomation), stained with hematoxylin, dehydrated, cleared, and mounted.

Migration assay. Migration assays were performed by assessment of the ability of cells to migrate toward 3T3-conditioned medium using $6.5-\mathrm{mm}$ Costar Transwell chambers ( $8 \mu \mathrm{m}$ pore size; Corning Inc.). Where indicated, Transwell membranes were coated on both surfaces with $20 \mu \mathrm{g} / \mathrm{ml}$ laminin-1 (Roche Diagnostics Corp.) or $50 \mu \mathrm{g} / \mathrm{ml}$ human cellular fibronectin (SigmaAldrich) by immersion overnight at $4{ }^{\circ} \mathrm{C}$, before aspiration and 3 washes in PBS. LIM 1863 organoids were treated with TNF- $\alpha$ and TGF- $\beta$ to induce an EMT and added to the upper chambers, and 3T3-conditioned medium was added to the lower wells of the chambers to induce chemotaxis. For antibody-inhibition studies, cells were seeded in medium containing either no antibody, anti- $\beta 6$ (10D5), or an isotype-matched control IgG antibody $(100 \mu \mathrm{g} / \mathrm{ml})$. After 3 days, cells were removed from the upper face of the filters using cotton swabs, and the cells that had migrated to the lower surface were fixed in methanol. Filters were mounted onto microscope slides using VECTASHIELD mounting medium with DAPI (Vector Laboratories Inc.), and migration was quantified by visual counting using fluorescence microscopy. Assays were carried out in triplicate, and the results presented are the means of 8 random fields from each well.

TGF- $\beta$ bioassay. In 96-well plates, LIM 1863 cells were cultured in the presence and absence of TNF- $\alpha$ and TGF- $\beta$ for 24 hours (in $100 \mu \mathrm{l}$ of RPMI plus 5\% FCS). After 3 gentle washes with DMEM containing 10\% FCS, $2 \times 10^{4}$ reporter cells (17) were seeded onto the LIM 1863 cells in $100 \mu \mathrm{l}$ per well in the absence or presence of the $\beta 6$-blocking antibody 
3G9 $(30 \mu \mathrm{g} / \mathrm{ml})$ or isotype-matched control (CS $\beta 6)$. Cells were cultured for an additional 16-20 hours, and lysates were assayed for luciferase activity as previously described (16).

Statistical methods. Kaplan-Meier method was used to estimate median survival, 5-year survival, and 10-year survival along with their $95 \%$ confidence intervals. Log-rank test was used to examine the differences in the overall survival between groups. The proportional hazard ratio model was used to obtain more precise estimates of the effects of protein expression on survival by adjusting for the effects of age, gender, tumor type, and cancer stage. In all analyses, only disease-related deaths were treated as events.

\section{Acknowledgments}

This work was supported by NIH grants CA 80789 and CA107548 (to A.M. Mercurio) and by the Harvard Digestive Diseases Center (NIH grant DK34854; to R.C. Bates). We wish to thank Jeffrey Goldsmith (Beth Israel Deaconess Medical Center), David Rimm (Yale University, New Haven, Connecticut, USA), and Shelia Violette (Biogen Idec, Cambridge, Massachusetts, USA) for invaluable contributions to this manuscript by way of reagents, techniques, and expertise.

Received for publication August 26, 2004, and accepted in revised form November 30, 2004.

Address correspondence to: Richard C. Bates, Department of Pathology, Division of Cancer Biology and Angiogenesis, Beth Israel Deaconess Medical Center, Research North, Room 220, 99 Brookline Avenue, Boston, Massachusetts 02215, USA. Phone: (617) 667-2816; Fax: (617) 975-5531; E-mail: rbates@caregroup.harvard.edu.
1. Hay, E.D. 1995. An overview of the epitheliomesenchymal transformation. Acta Anat. 154:8-20.

2. Savagner, P. 2001. Leaving the neighborhood: molecular mechanisms involved during epithelialmesenchymal transition. Bioessays. 23:912-923.

3. Theiry, J.P. 2003. Epithelial-mesenchymal transitions in development and pathologies. Curr. Opin. Cell Biol. 15:740-746.

4. Oft, M., et al. 1996. TGF-beta1 and Ha-Ras collaborate in modulating the phenotypic plasticity and invasiveness of epithelial tumor cells. Genes Dev. 10:2462-2477.

5. Oft, M., Heider, K.H., and Berg, H. 1998. TGF $\beta$ signaling is necessary for carcinoma cell invasiveness and metastasis. Curr. Biol. 8:1243-1252.

6. Bhowmick, N.A., et al. 2001. Transforming growth factor- $\beta 1$ mediates epithelial to mesenchymal transdifferentiation through a Rho-A-dependent mechanism. Mol. Biol. Cell. 12:27-36.

7. Thiery, J.P. 2002. Epithelial-mesenchymal transitions in tumour progression. Nat. Rev. Cancer. 2:442-454.

8. Hynes, R.O. 1992. Integrins: versatility, modulation, and signaling in cell adhesion. Cell. 69:11-25. 9. Ruoslahti, E. 1991. Integrins. J. Clin. Invest. 87:1-5.

10. Bates, R.C., and Mercurio, A.M. 2003. Tumor necrosis factor- $\alpha$ stimulates the epithelial to mesenchymal transition of human colonic organoids. Mol. Biol. Cell. 14:1790-1800.

11. Bates, R.C., Buret, A., van Helden, D.F., Horton, M.A., and Burns, G.F. 1994. Apoptosis induced by inhibition of intercellular contact. J. Cell Biol. 125:403-415.

12. Bates, R.C., et al. 2003. Flt-1-dependent survival characterizes the epithelial-mesenchymal transition of colonic organoids. Curr. Biol. 13:1721-1727.

13. Oikawa, T., and Yamada, T. 2003. Molecular biology of the Ets family of transcription factors. Gene. 303:11-34

14. Busk, M., Pytela, R., and Sheppard, D. 1992. Characterization of the integrin $\alpha v \beta 6$ as a fibronectin- binding protein. J. Biol. Chem. 267:5790-5796.

15. Lehmann, K., et al. 2000. Raf induces TGF $\beta$ production while blocking its apoptotic but not invasive responses: a mechanism leading to increased malignancy in epithelial cells. Genes Dev. 14:2610-2622.

16. Munger, J.S., et al. 1999. The integrin $\alpha v \beta 6$ binds and activates latent TGF $\beta 1$ : a mechanism for regulating pulmonary inflammation and fibrosis. Cell. 96:319-328.

17. Abe, M., et al. 1994. An assay for transforming growth factor-beta using cells transfected with a plasminogen activator inhibitor-1 promoterluciferase construct. Anal. Biochem. 216:276-284.

18. Breuss, J.M., Gillett, N., Lu, L., Sheppard, D., and Pytela, R. 1993. Restricted distribution of integrin beta 6 mRNA in primate epithelial tissues. J. Histochem. Cytochem. 41:1521-1527.

19. Breuss, J.M., et al. 1995. Expression of the $\beta 6$ integrin subunit in development, neoplasia and tissue repair suggests a role in epithelial remodeling. J. Cell Sci. 108:2241-2251.

20. Zambruno, G., et al. 1995. Transforming growth factor-beta 1 modulates beta 1 and beta 5 integrin receptors and induces the de novo expression of the alpha $v$ beta 6 heterodimer in normal human keratinocytes: implications for wound healing. J. Cell Biol. 129:853-865.

21. Pilewski, J.M., Latoche, J.D., Arcasoy, S.M., and Albelda, S.M. 1997. Expression of integrin cell adhesion receptors during human airway epithelial repair in vivo. Am. J. Physiol. 273:L256-L263.

22. Hamidi, S., et al. 2000. Expression of alpha(v)beta6 integrin in oral leukoplakia. Br.J. Cancer. 82:1433-1440.

23. Regezi, J.A., Ramos, D.M., Pytela, R., Dekker, N.P., and Jordan, R.C.K. 2002. Tenascin and $\beta 6$ integrin are overexpressed in floor of mouth in situ carcinomas and invasive squamous cell carcinomas. Oral Oncol. 38:332-336.

24. Pittet, J.F., et al. 2001. TGF- $\beta$ is a critical mediator of acute lung injury. J. Clin. Invest. 107:1537-1544.
25. Morris, D.G., et al. 2003. Loss of integrin alpha(v)beta6-mediated TGF-beta activation causes Mmp-12-dependent emphysema. Nature. 422:169-173.

26. Bates, R.C. 2003. Gastrointestinal matrix, organization and significance. In Encyclopedia of gastroenterology. L.R. Johnson, editor. Elsevier Science. San Diego, California, USA. 219-223.

27. Prieto, A.L., Edelman, G.M., and Crossin, K.L. 1993. Multiple integrins mediate cell attachment to cytotactin/tenascin. Proc. Natl. Acad. Sci. U. S. A. 90:10154-10158.

28. Kemperman, H., Driessens, M.H., La Riviere, G., Meijne, A.M., and Roos, E. 1995. Adhesion mechanisms in liver metastasis formation. Cancer Surv. 24:67-79.

29. Weinreb, P.H., et al. 2004. Function-blocking integrin $\alpha v \beta 6$ monoclonal antibodies: distinct ligand-mimetic and non-ligand mimetic classes. J. Biol. Chem. 279:17875-17887.

30. Whitehead, R.H., Jones, J.K., Gabriel, A., and Lukies, R.E. 1987. A new colon carcinoma cell line (LIM 1863) that grows as organoids with spontaneous differentiation into crypt-like structures in vitro. Cancer Res. 47:2683-2689.

31. National Center for Biotechnology Information. http://www.ncbi.nlm.nih.gov/.

32. Oettgen, P., et al. 1996. Characterization of NERF, a novel transcription factor related to the Ets factor Elf-1. Mol. Cell. Biol. 16:5091-5106.

33. Farr, A., and Roman, A. 1992. A pitfall of using a second plasmid to determine transfection efficiency. Nucleic Acids Res. 20:920.

34. Dube, A., Akbarali, Y., Sato, T.N., Libermann, T.A., and Oettgen, P. 1999. Role of the Ets transcription factors in the regulation of the vascular-specific Tie2 gene. Circ. Res. 84:1177-1185.

35. Rimm, D.L., Camp, R.L., Charette, LA., Olsen, D.A., and Provost, E. 2001. Amplification of tissue by construction of tissue microarrays. Exp. Mol. Pathol. 70:255-264. 